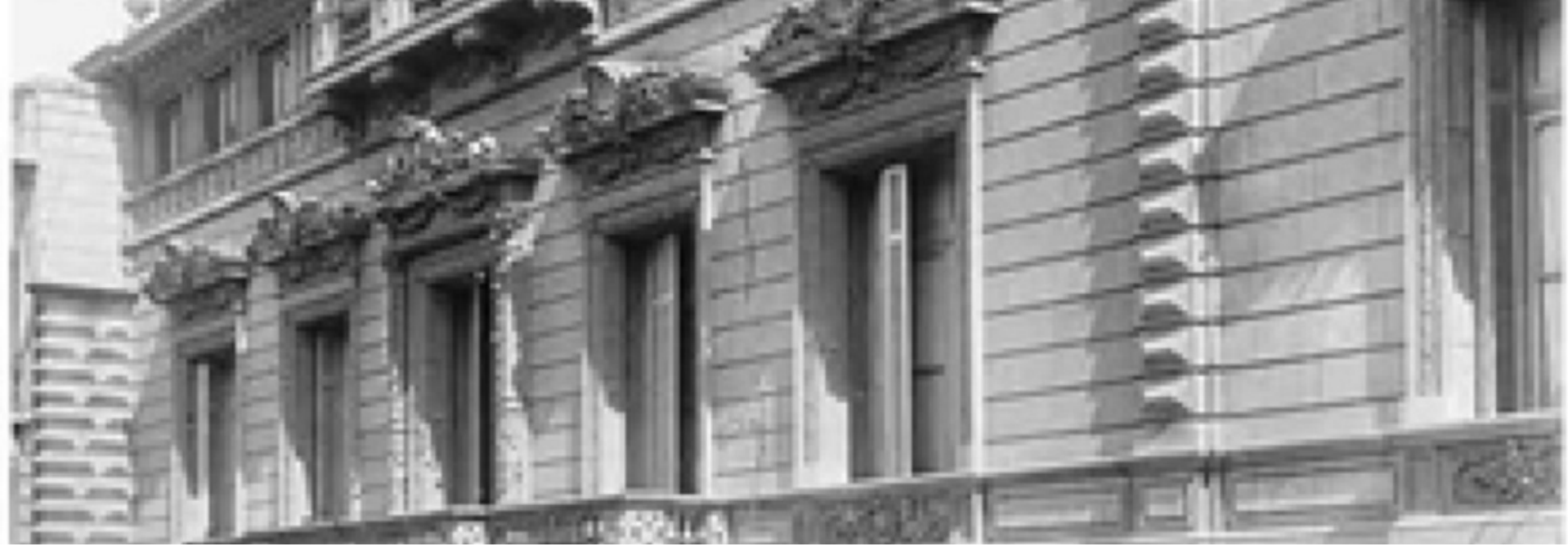

\title{
History of the German philology in Argentina (chapter 5) - GESCHICHTE DER ARGENTINISCHEN GERMANISTIK
}

Authors: Lila Bujaldon de Esteves

Submitted:

2. July 2018

Published:

Volume:

19. July 2018

Issue:

Affiliation:

Languages:

Keywords:

DOI:

Abstract:

\section{5}

6

CONICET (Nationaler Rat für wissenschaftliche und technologische Forschung), UNCUYO (Universidad Nacional de Cuyo)

\section{German}

History, Authors, Literature, Argentina, Germany

10.17160/josha.5.6.440

This study of the history of German philology in Argentina describes and analyses the period of time between the Revolution of May 1810, which means after the independence of Argentina from Spain, and the 1950s. The study has its focus on the history of the degree program of the University of Buenos Aires. We are going to read about the first professors of German literature at the University in chapter four, five and six. This chapter is about Albert Haas 
Argentum virtus robur et studium - La virtud argentina es la fuerza y el estudio -

Die argentinische Tugend ist die Kraft und das Studium

\section{GESCHICHTE DER ARGENTINISCHEN GERMANISTIK}

\section{Lila Bujaldon de Esteves}

\section{Affiliation: CONICET (Nationaler Rat für wissenschaftliche und technologische Forschung), UNCUYO (Universidad Nacional de Cuyo)}

\section{$\underline{\text { Abstract }}$}

Diese Studie zur Geschichte der Germanistik in Argentinien befasst sich mit der Zeitspanne zwischen der Mai-Revolution 1810 -d.h. der Zeit nach der Unabhängigkeit von Spanienund den 50er Jahren. Die Studie setzt das Augenmerk auf die Geschichte des Studiengangs an der Universität Buenos Aires.

Als erstes wird die Wissenschaftsgeschichte der Germanistik dargestellt. Daran anschließend wird die Entwicklung der Fremdsprachenlehre -u.a. der deutschen Sprachevon der Kolonialzeit bis zu den ersten Jahren des 20. Jahrhunderts untersucht. Ein Kapitel ist der lokalen Bezeichnung der Germanistik gewidmet: „Literatur des nördlichen Europa“. Auch die Biographien der ersten Professoren Deutscher Literatur an der Universität Buenos Aires -Mauricio Nirenstein, Albert Haas und Juan Carlos Probst- werden in weiteren Kapiteln eingehend untersucht. Das Schlusskapitel stellt darüber hinaus die Arbeit von Werner Bock, Ilse M. de Brugger und Gúnter Ballin vor. Alle haben während des Deutschen Exils 1933 in Argentinien Zuflucht gefunden und gaben die Deutsche Literatur in der akademischen und intellektuellen Szene bekannt. 
Argentum virtus robur et studium - La virtud argentina es la fuerza y el estudio -

Die argentinische Tugend ist die Kraft und das Studium

\section{Kapitel V}

Albert Haas (1873-1930) als kultureller Mittler zwischen Deutschland und Argentinien nach dem Ersten Weltkrieg. Germanistische Leistungen und kulturpolitische Einstellung.

Der Präsenz und Tätigkeit von Albert Haas in Argentinien bilden den Übergang zwischen einer ersten "dilettantischen" Phase und der darauffolgenden Konsolidierung der Germanistik als einer autonomen Disziplin an der Universität Buenos Aires. Im Jahr 1928 veranstaltete Albert Haas einen Vortragszyklus an der Philosophischen Fakultät der Universität Buenos Aires über die Geschichte der neueren deutschen Literatur. In der Folge dieses Kurses wurde sein Lehrbuch: Historia de la literatura alemana veröffentlicht. Auf diese Weise entstand im 20. Jahrhundert die erste auf Spanisch verfasste Geschichte der deutschen Literatur, die sofort unter den Studenten Verbreitung fand und in den wichtigsten akademischen Publikationen rezensiert wurde. Im gleichen Jahr 1928 las Mauricio Nierenstein, der Professor für nordeuropäische Literaturen, zum ersten Mal im Rahmen dieses Lehrstuhles über Englische und nicht Deutsche Literatur- anders als in den sieben Jahren davor. Darum kann man vermuten, dass Albert Haas im Grunde die germanistischen Vorlesungen Nierenstein durch seine Vorträge und akademischen Veröffentlichungen ersetzt hat.

Haas hatte in Berlin Philosophie, Nationalökonomie und Germanistik studiert und zwar in einer Zeit, als die Germanisten dort eine wichtige gesellschaftliche Rolle spielten. Sein Germanistikprofessor, Erich Schmidt, war in den Zeitungen und im Berliner kulturellen Leben eine fortführende Persönlichkeit. Nach diesem Vorbild entwickelte sich Haas als Dozent im Ausland. Sechs Jahre lang lehrte er Germanistik im Westen der Vereinigten Staaten. Nach seiner Rückkehr nach Deutschland widmete er sich dem Wirtschaftsjournalismus und der Lehre dieser Disziplin in Berlin und Hamburg. 
Argentum virtus robur et studium - La virtud argentina es la fuerza y el estudio -

Die argentinische Tugend ist die Kraft und das Studium

Der Erste Weltkrieg brachte schwere Erfahrungen für Haas, der als Journalist für das neutrale Ausland berichtete. Seine damaligen Erlebnisse führten ihn zu der Erkenntnis, dass die deutsche Kultur im spanischen Sprachraum aufgrund einer falschen auswärtigen Politik sehr wenig verbreitet war. Die Isolierung Deutschlands während der Kriegszeit betrachtete er als eine Folge dieser Politik. Nach dem Krieg wurde er von der transozeanischen Nachrichtengesellschaft nach Argentinien entsandt und schrieb dort wöchentlich für die deutschen Zeitungen, vor allem über die argentinische Gegenwart. Er war überzeugt, dass eine Kulturarbeit dem gegenseitigen Kennenlernen der Partner entspricht. Deswegen versuchte er dem deutschen Publikum Geschichte, Literatur sowie die wirtschaftliche, soziale und politische Entwicklung Argentiniens nahe zu bringen. Gleichzeitig leitete er in Argentinien die Zeitschrift Phoenix, die deutsche Forschungen über das Gastland und andere südamerikanische Regionen verbreitete. Dort veröffentlichte er auch eigene Übersetzungen von Werken neuerer argentinischer Autoren, die die damalige soziale Einwanderungsproblematik des Landes spiegelten, wie etwa die des Dramatikers Florencio Sánchez und des Romanciers Manuel Gálvez.

Mit mehreren argentinischen Historikern teilte er die Überzeugung, dass die spanische Kultur grundlegend für die Bildung einer argentinischen Identität gewesen sei. Selbst hinsichtlich der Unabhängigkeitsideen sei Frankreich nicht die einzige Übermittlerin gewesen, vielmehr hätten gewisse Institutionen der mittelalterlichen spanischen Städte, die sogenannten "fueros", d.h. Sonderrechte, dabei eine Rolle gespielt. Damit versucht Haas, den Einfluss Spaniens auf die argentinische Kultur $\mathrm{zu}$ betonen und so indirekt dazu beizutragen, die Abhängigkeit von der französischen Kultur zurückzudrängen. Aus dem großen Einfluss Frankreichs in Argentinien leitete Haas übrigens die Tatsache ab, dass die deutsche Literatur überwiegend durch französische Übersetzungen vermittelt war, was er in Bezug auf die deutsch-argentinischen Kulturbeziehungen für schädlich hielt. 
Argentum virtus robur et studium - La virtud argentina es la fuerza y el estudio -

Die argentinische Tugend ist die Kraft und das Studium

Albert Haas brachte eine von ihm ins Spanische übersetzte Anthologie deutscher Lyrik heraus. Auch in seiner Historia de la literatura alemana moderna fügte er selbstübersetzte Prosa- und Poesiefragmente ein, die als Beitrag zur Verbreitung unbekannter Texte im spanischen Sprachraum betrachtet werden können. Vor der Veröffentlichung seiner Deutschen Literaturgeschichte hatte Haas mehr als sieben Jahre lang an diesem Stoff gearbeitet und manche ihrer Kapitel schon in argentinischen Zeitschriften vorab drucken lassen. In der Einführung zu dieser Literaturgeschichte resümierte Haas die Entwicklung der deutschen Literatur bis zum Jahre 1770. In den folgenden 25 Kapitel hob er die Einheit der deutschen Literatur hervor, die auf der gemeinsamen Sprache und auf den kämpferischen und völkischen Zügen beruhe. Nachdem das Lehrbuch vergriffen war, wurde es nicht wieder aufgelegt, u.a. weil der Direktor des 1937 gegründeten Instituto de Estudios Germánicos es für ausländisches Publikum als parteiisch und irreführend betrachtete. In seinen letzten unveröffentlichten Arbeiten beschäftigte sich Albert Haas mit den soziologischen Ideen des späten Goethe.

Albert Haas hinterließ eine erstaunlich lange Reihe journalistischer Arbeiten, die in der Zeit seines Argentinienaufenthalts (1920-1930) vor allem in der Kölnischen Zeitung gedruckt wurden. Seine Themen umfassten Wirtschaft, Politik, Kultur und internationale Beziehungen Argentiniens in Bezug auf deutsche Interessen. Zusätzlich arbeitete er als Wirtschaftsbeirat an der damaligen "Deutschen Gesandtschaft", d.h. der Vorgängerin der Deutschen Botschaft in Buenos Aires, wo er für Einwanderungsfragen verantwortlich war. 
Argentum virtus robur et studium - La virtud argentina es la fuerza y el estudio -

Die argentinische Tugend ist die Kraft und das Studium

\section{Ausgewählte Bibliographie}

Selbständige Publikationen (1919-1930)

$\underline{\text { Bücher }}$

Argentinien. Stuttgart: Perthes“ Kleine Völker- imd Länderkunde zum Gebrauch im praktischen Leben”, 10. Band, 1923.

Flores de la poesía alemana. Seleccionadas y traducidas por Albert Haas y Federico More. T. 1. Buenos Aires: Editora internacional, 1924.

Historia de la literatura alemana moderna. Buenos Aires: Imprenta de la Universidad, 1928.

\section{$\underline{\text { Übersetzungen }}$}

Jacinto Benavente, Der tugendhafte Glücksritter. Die frohe Stadt des Leichtsinns. Der letzte Menuett. Zus. M. Enrique Dominguez Rodiño u. Max Brausewetter. München 1919; Ángel Ganivet, Spaniens Weltanschauung und Weltstellung. München 1921; Manuel Gálvez, Nacha Regules, Argentinischer Roman. Berlin 1922; Novalis, Himnos a la noche. Zus. M. Federico More. In: Fénix 4 (1924) 67-72. Georg Christoph Lichtenberg, Aforismos.

Buenos Aires 1924; Florencio Sánchez, Einwanderer. Ein Schauspiel in 4 Aufzügen. Buenos Aires 1925.

\section{$\underline{\text { Aufsätze und Publikationsorgane }}$}

Zahlreiche Ausätze in den folgenden erwähnten Zeitungen und Zeitschriften: Nosotros. Revista mensual de Letras, Arte, Historia, Filosofía y Ciencias Sociales; Phoenix. Zeitschrift für deutsches Geistesarbeit in Südamerika; Fénix; Buenos Aires HandelsZeitung; Deutsche La Plata Zeitung; Zeitschrift für Politik Berlin; National- Zeitung Berlin; Hamburger Femdenblatt; Wirtschaftsdienst Hamburg; Der Auslanddeutsche. 
Argentum virtus robur et studium - La virtud argentina es la fuerza y el estudio -

Die argentinische Tugend ist die Kraft und das Studium

Halbmonatsschrift des deutschen Ausland- Institutes Stuttgart; Kölnische Zeitung; Vossische Zeitung.

\section{$\underline{\text { Über Albert Haas }}$}

Biographisches Handbuch des deutschen Auswärtigen Dienstes 1871-1945. Bd.2: G-K. Bearb. Gerhard Keiper.Martin Kröger. Hrg. vom Auswärtigen Amt durch den Historischen Dienst (Maria Keipert und Peter Grupp).

Fittbogen, Gottfried. “Dr. Albert Haas zum Gedächtnis”. Mit einer Liste seiner Publikationen. Ibero-Amerikansiches Archiv 5, 2, 1931, 125-137.

Bujaldon de Esteves, Lila. “Albert Haas”. Internationales Germanistenlexikon 1800-1950. Hrg. Christoph König. Berlin: De Gruyter, 2003, 645-646.

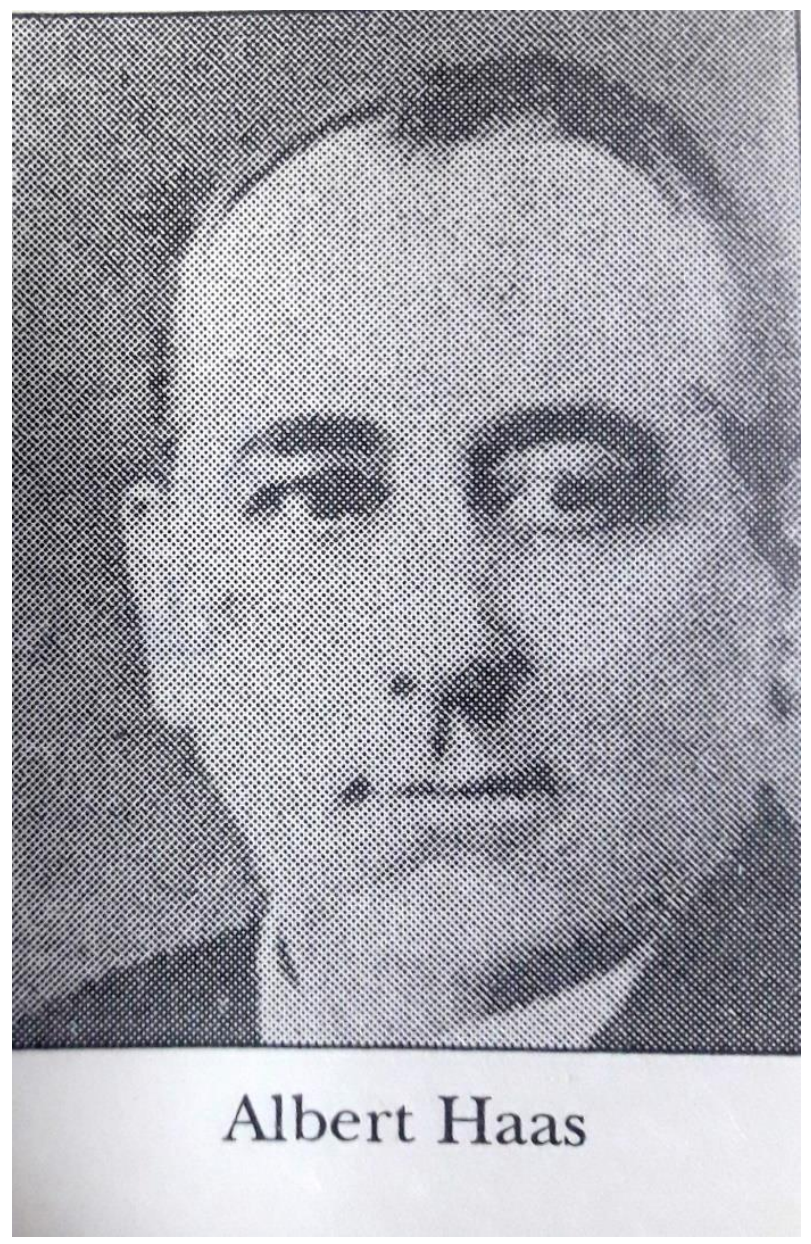

